\title{
Empirical Evaluation of Throwing Method to Move Object for Long Distance in 3D Information Space on Mobile Device
}

\author{
Yu Shibuya, Keiichiro Nagatomo, Kazuyoshi Murata, \\ Itaru Kuramoto, and Yoshihiro Tsujino \\ Kyoto Institute of Technology \\ Matsugasaki, Sakyo-ku, Kyoto 606-8585, Japan \\ shibuya@kit.ac.jp
}

\begin{abstract}
In our previous work, a throwing method to move an object for long distance in 3D information space on a mobile device was proposed. With the method, as we throw the object to move it far away in the real world, we can throw the virtual object in 3D information space. This simple throwing method was improved by adding following three functions. They were real time adjusting the direction of moving object, moving viewpoint to follow the thrown object, and initializing viewpoint after the movement. The purpose of this paper is to examine the performance of the improved throwing method to move the object for long distance in 3D information space. From the experiment, it is found that the improved throwing method is efficient to move the object for long distance in 3D information space on mobile devices.
\end{abstract}

Keywords: mobile interaction, 3D information space, throwing method, human computer interaction.

\section{Introduction}

In these days, many people carry their own mobile devices such as PDAs or mobile phones. Modern mobile devices have power to process and display 3D information space. 3D information space is usable for various purposes, such as showing complex structures or representing some realistic objects. However, there is no suitable interaction method to operate the object in 3D information space on mobile devices.

In our former work [1], we proposed a new interaction method, named as Handy Window. In that work, it was found that the hand gesture behind the mobile device was usable to move or rotate the virtual object (Fig. 1). However, in order to move the object far away, users are suffering from physical fatigue or the task performance is decreased because they should move the mobile device or their hand widely. In order to avoid such long movement, we then proposed a novel object moving method with throwing gesture, named as throwing method [2] (Fig. 2). With the method, users can not only throw the object in 3D information space but also adjust the direction of moving object as they want, follow the thrown object by moving viewpoint, and initialize viewpoint easily after the movement. 
From the preliminary experiment, it was found that the proposed method reduced the physical fatigue of the user but there was no significant improvement of the performance. Because the previous experiment treated the short movement only, it might be difficult to find the difference between the throwing method and non-throwing method. This paper focuses on evaluating the performance of the improved throwing method to move the object for long distance in 3D information space.

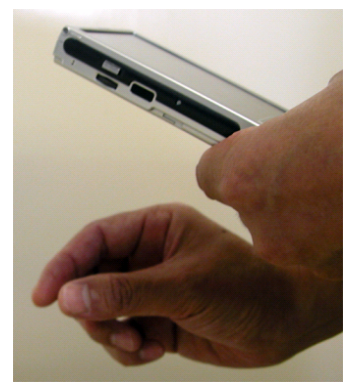

Fig. 1. Concept of Handy Window. The user can move or rotate the virtual object with the hand gesture behind the mobile device.
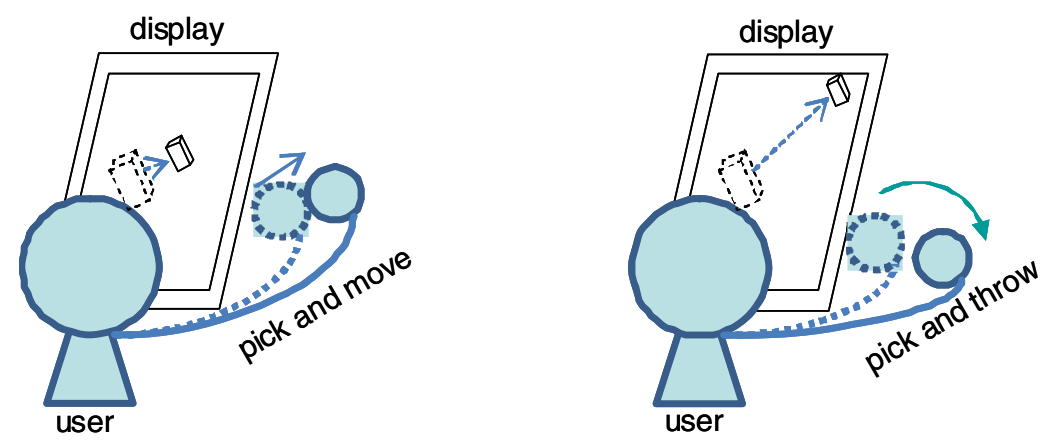

Fig. 2. Two object moving methods. With the pick and move method (left), the user can move the object for short distance with changing his/her hand position. With the throwing method (right), the user can move the object for long distance with throwing gesture.

\section{Throwing Method}

As mentioned above, the throwing method was proposed in our previous work [2]. With the method, as we throw the object to move it far away in the real world, we can throw the virtual object in 3D information space in order to move it for long distance. In the method, a user picks up an object with his/her fingers firstly, then moves his/her hand to the desired direction with a certain velocity, and releases the object. After that, the object begins to fly to the target position. When the user makes a picking action again, the object stops to fly. Then the user can adjust the location of the 
object by moving his/her hand. Finally, when he/she releases the object without moving his/her hand, the object is fixed there.

However, with above simple throwing method, it is sometimes difficult to move the object far away. As we experienced in the real world, it is difficult to throw the object to the desired direction accurately. Furthermore, because the thrown object is getting smaller on the display as it moved, it is difficult to grasp the object location accurately. In order to tackle the first problem, the user is allowed to adjust the direction of the flying object by his/her finger gesture (Fig. 3). For the second problem, the view point follows the thrown object and the size of object is not changed until it is fixed (Fig. 4). Furthermore, the user can get the view point back with simple hand gesture (Fig. 5). In this paper, we call this method improved throwing method to distinguish with simple one.

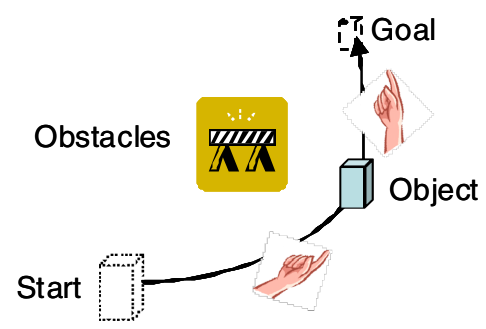

Fig. 3. Adjustment of direction. The user can change the direction of moving object with changing his/her finger direction.

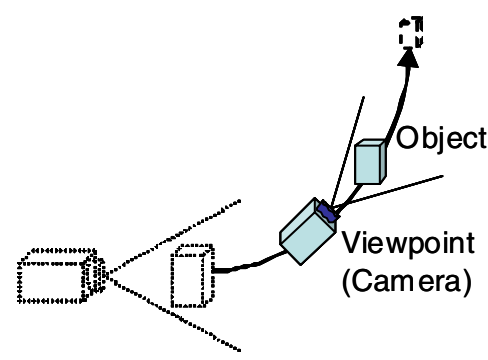

Fig. 4. View control. Camera follows the object to show it in moderate size on the display.
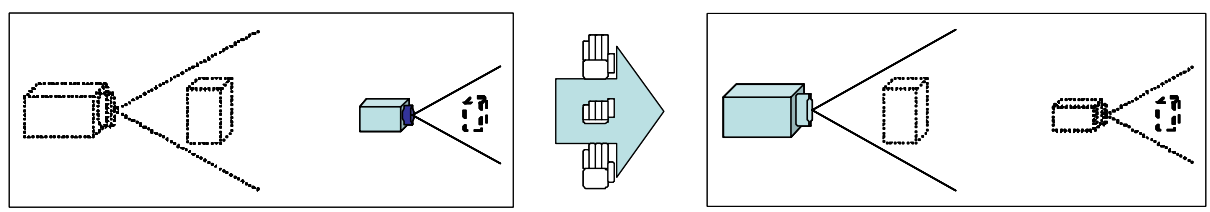

Fig. 5. View initialization. After object movement (left), camera view point is initialized (right) with beckoning hand gesture (center). 


\section{Experiment}

The purpose of the experiment is to examine the performance of the improved throwing method to move the object for long distance in $3 \mathrm{D}$ information space.

\subsection{Compared Moving Methods}

Three kinds of object moving method are evaluated in the experiment. They are the improved throwing method, the simple throwing method, and the non-throwing method. The non-throwing method does not allow the user to throw the object. The user must move his/her hand or body to move the picked object. We expected that the efficiency of both throwing methods was getting better than that of non-throwing method as the distance to move was getting longer. Furthermore, the improved throwing method should be better than simple one.

\subsection{Prototype System for Experiment}

A prototype system was constructed for the experiment as shown in Fig. 6. It consists of a motion tracker for tracking position and orientation of the mobile device and participant's hand, and a data glove for detecting participant's hand gesture. With the limit of the detectable distance of the motion tracker, participants could not be longer than one meter away from the transmitter of the tracking system. With this reason, non-throwing method, which needs participant's movement, is evaluated in short distance movement only.

In the prototype system, sensed data of the motion tracker and that of the data glove are processed by a desktop PC. The PC also makes a visual feedback for the mobile device.

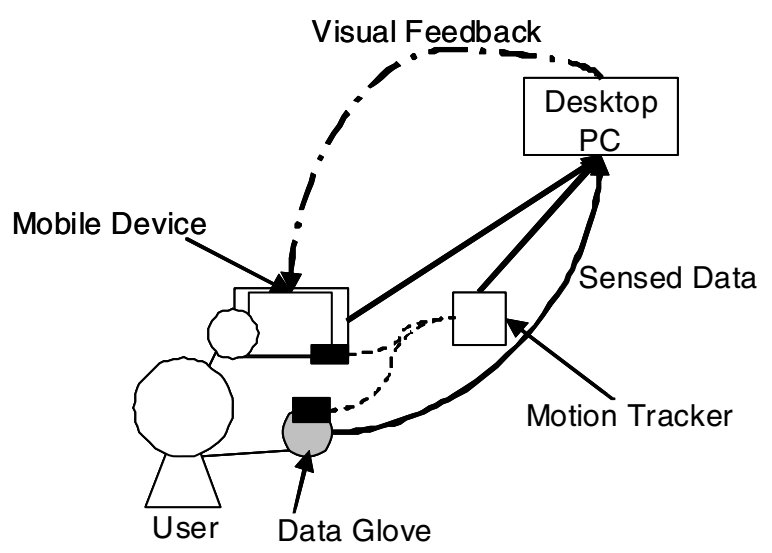

Fig. 6. System configuration of the experiment 


\subsection{Procedure}

Twelve participants were recruited from our research laboratory. In the experiment, each participant was asked to pick up the object at the starting point and move it the target point. The distance between the staring point and target one was set at $0.5 \mathrm{~m}$, $1 \mathrm{~m}, 1.5 \mathrm{~m}, 3 \mathrm{~m}$, and $5 \mathrm{~m}$ respectively. In the experiment, task completion time and error rate were measured. Subjective evaluation was also done to evaluate the intuitiveness, ease of learning to use, and the level of fatigue of each moving method. Fig. 7 shows a snap shot of an experiment.

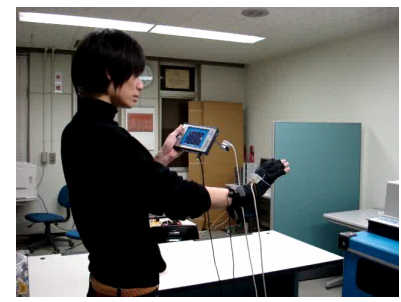

(a) Overview

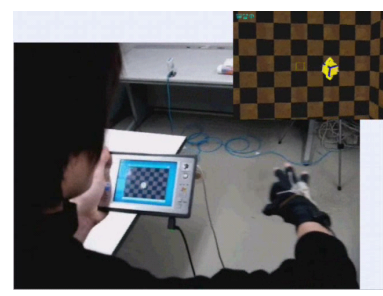

(b) View over the user's shoulder

Fig. 7. A snapshot of experiment

\section{Results and Discussion}

\subsection{Task Completion Time}

From the experiment, as shown in Fig. 8, it was found that the task completion time with non-throwing method was significantly shorter than the simple throwing method

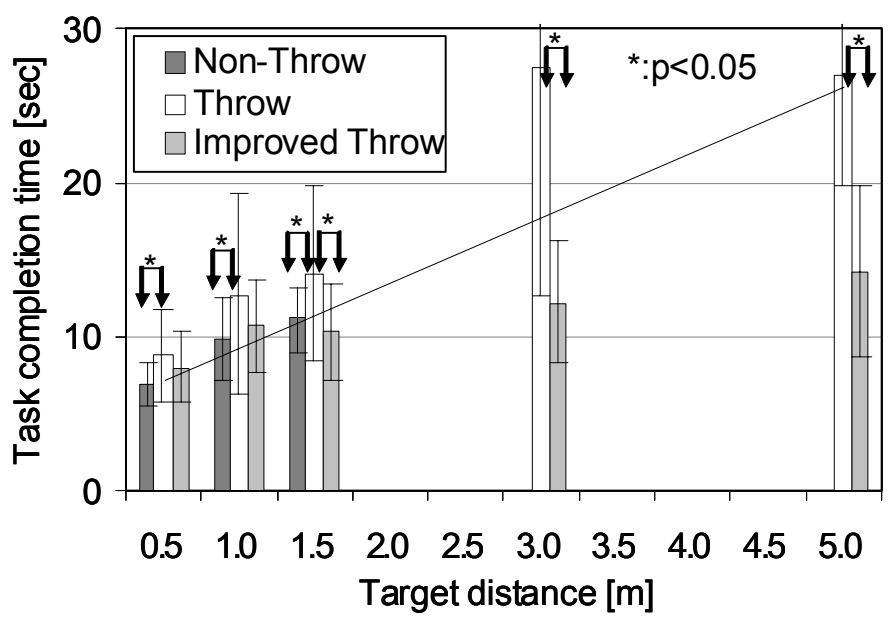

Fig. 8. Task completion time 
$(\mathrm{p}<0.05)$ while the distance for move was less than or equal to $1.5 \mathrm{~m}$. However, it was linearly getting longer as the distance was increased. The task completion time of simple throwing method was also getting longer with the distance was increased. On the other hand, the task completion time of the improved throwing method was not so changed while the distance was increased. The results show that the improved throwing method has better performance to move the object for long distance than other methods. With the improved throwing method, participants can adjust the direction of the object while moving and they can also easily set the object at the target point because both object and target has moderate view size on the display. Furthermore, in order to complete the task, they can quickly initialize the viewpoint after the movement with simple hand gesture. These are why the improved throwing method is more efficient than others.

\subsection{Error Rate}

The result of error rate is shown in Fig. 9. From this figure, it is found that there was no significant deference but the error rate of simple throwing method increased as the target distance became long. However, talking about the improved throwing method, the error rate of it was not changed so much. With the simple throwing method, it was difficult to set the object to the far target position because the target size was so small on the display. With the improved method, the view camera in 3D information space follows the moving object ant the user can see the target in reasonable size on the display when the object closes to it. This made the error rate of the improved throwing method lower than that of simple one.

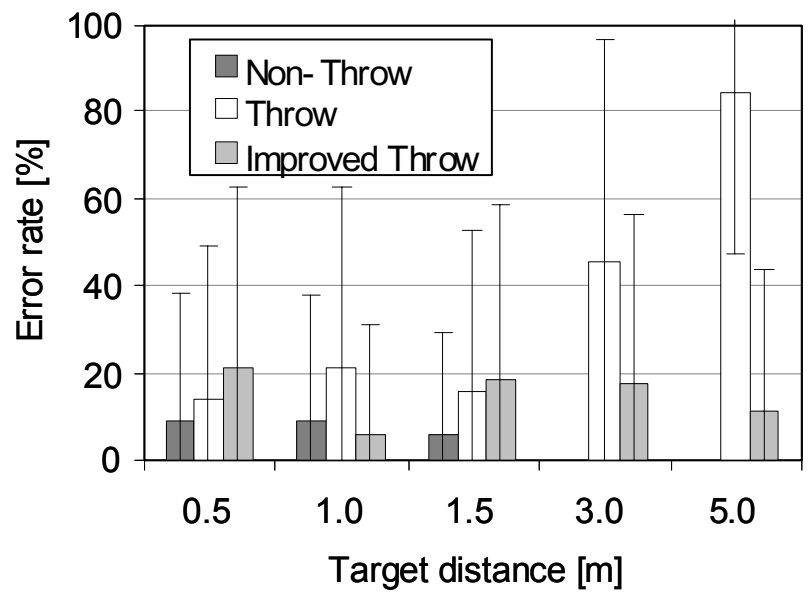

Fig. 9. Error rate

\subsection{Subjective Evaluation}

Fig. 10 shows the result of subjective evaluation. From the subjective evaluation, followings were found. Firstly, the non-throwing method was significantly more intuitive 


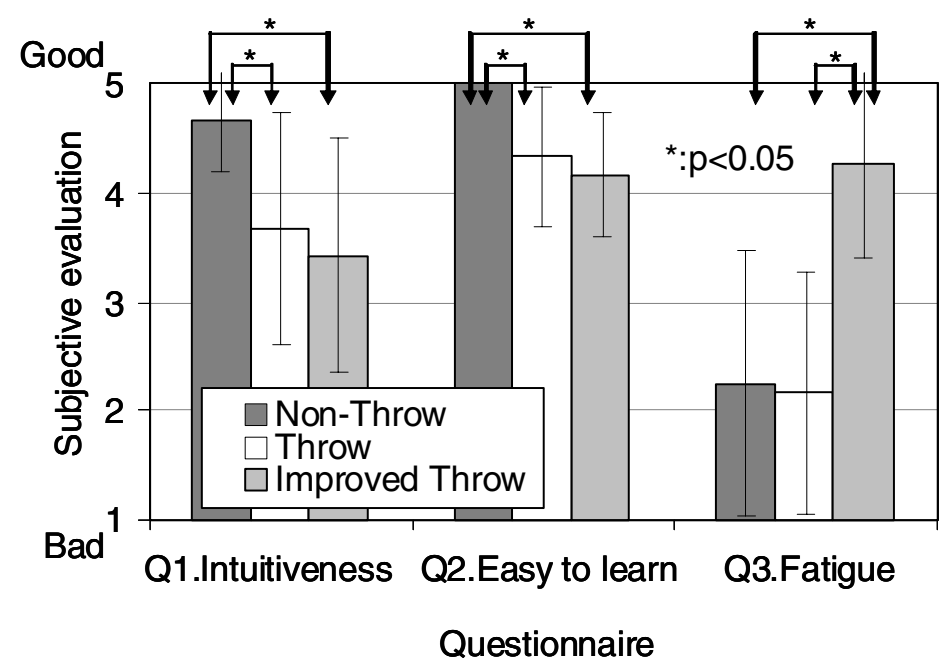

Fig. 10. Subjective evaluation

than others $(p<0.05)$ but the intuitiveness of both throwing method was moderate. Secondly, all three methods were easy to learn. From these results, most user might be soon familiar with throwing virtual object method while they were not so familiar with it until the experiment. Finally, the level of fatigue of the improved throwing method was significantly better, namely lower, than others $(\mathrm{p}<0.05)$. With the improved throwing method, participants did not move themselves so much while they must move so much with non-throwing method or re-throw so many times with simple throwing method.

\section{Related Work}

Throwing action has been used to move objects across larger distance on wall size screen [3], [4], but the action has not been used for the object in 3D information space on mobile devices.

Gilbertson et al. [5] explored a tilt interface for a 3D graphics first-person driving game and compared it with a traditional phone joypad interface experimentally. They showed that the tilt interface was experienced as fun and attractive to players. They focused on navigation in virtual 3D information space. This paper focuses on not only the navigation but also the manipulation of the virtual object in $3 \mathrm{D}$ information space on mobile phones.

\section{Conclusion}

In this paper, the empirical evaluation of the improved throwing method was done. From the experiment, it is found that the improved throwing method is efficient to move the object for long distance in 3D information space on mobile device. Furthermore, it is also found that the additional three functions, adjusting the direction 
while moving, moving viewpoint to follow the thrown object, and initializing the viewpoint after movement, are effective to improve the task performance.

\section{References}

1. Shibuya, Y., Taniguchi, N., Kuramoto, I., Tsujino, Y.: Handy Window: An Interface for Intuitive Interaction of My Portable Information Terminal and the Other Ubiquitous Devices. In: Proc. HCI International 2005 (CD-ROM), vol. 5 (2005)

2. Nagatomo, K., Murata, K., Kuramoto, I., Shibuya, Y., Tsujino, Y.: Efficient Object Moving Method with Throwing Gesture in 3D Information Space on Mobile Device. In: Proc. Symposium on Mobile Interactions, pp. 53-58 (2008) (in Japanese)

3. Geissler, J.: Shuffle, Throw or Take It! Working Efficiently with an Interactive Wall. In: CHI 1998 conference summary on Human factors in computing systems, pp. 265-266 (1998)

4. Collomb, M., Hascoeet, M., Baudisch, P., Lee, B.: Improving Drag-and-Drop on Wall-size Displays. In: Proceedings of Graphics Interface 2005, pp. 25-32 (2005)

5. Gilbertson, P., Coulton, P., Chehimi, F., Vajk, T.: Using "Tilt" as an Interface to Control "No-Button" 3-D Mobile Games. Comput. Entertain. 6(3), 1-13 (2008) 